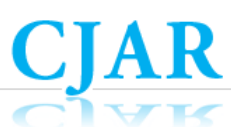

Canadian Journal of Action Research Volume 19, Issue 3, 2018, pages 53-72

\title{
CREATING PRODUCTIVE TENSIONS: CLINICIANS WORKING WITH PATIENTS AS PEER RESEARCHERS IN A COMMUNITY-BASED PARTICIPATORY RESEARCH STUDY OF THE LIVED EXPERIENCE OF HIV-ASSOCIATED NEUROCOGNITIVE DISORDER (HAND)
}

Francisco Ibáñez-Carrasco, Alex Terpstra, Sean Rourke, Aiko Yamamoto, Soo Chan Carusone, Rosalind Boltzer-Turje, Patrick McDougall, Claudia Medina, Kelly 0'Brien, Catherine Worthington

St. Michael's Hospital

\begin{abstract}
About 50\% of people living with HIV will develop HIV-associated neurocognitive disorder (HAND) during their lifetime, and we know that cognitive issues are a concern for people living with HIV. However, limited information exists regarding how HAND is managed and coped with, or how cognitive issues are discussed with others, including health care professionals. Following a community-based research approach, we conducted 25 interviews in 2016 aimed to (1) build the capacity of people living with HIV, (2) facilitate participant recruitment and data collection, (3) increase the validity and reliability of our data analysis results, and (4) facilitate knowledge transfer and exchange regarding HAND. After thorough training, we engaged a number of peer researchers living with HAND in the analysis and knowledge transfer and exchange (KTE) phases of the study. This engagement prompted a number of tensions between the clinicians and the peers that we learned to navigate and make productive. We conclude that it is possible to engage patients and providers only if careful attention, time and human resources are provided to navigating the emerging tensions. The outcomes of our study suggest that engaging an interdisciplinary team across multiple sites with PRAs is a valuable method for comprehensively exploring the lived experience of a complex chronic condition such as HAND.
\end{abstract}

KEYwORDS: Interdisciplinary Teams; human immunodeficiency viruses - associated neurocognitive disorder (HAND) 


\section{INTRODUCTION}

In resource-rich countries where HIV can be effectively managed through the consistent use of antiretroviral medications, people living with HIV (PLWHIV) are living longer with the health-related consequences of HIV (McDermott et al., 2017; Mendes, 2015; Tedaldi, Minniti, \& Fischer, 2015). About 50\% of PLWHIV will develop HIV-associated neurocognitive disorder (HAND) during their lifetime (Antinori et al., 2007; Heaton et al., 2010), and cognitive issues are a concern for people living with HIV (Cummins et al., 2018; Eaton, Craig, \& Wallace, 2017). However, limited information exists regarding how HAND is managed and coped with, or how cognitive issues are discussed with others, including health care professionals. To add to this literature, our community-based participatory research (CBPR) team (PLWHIV and HAND, clinicians, researchers, and trainees), conducted a qualitative study with PLWHIV, and produced information tools to raise awareness of HAND for PLWHIV, health care providers, and AIDS service organizations. We explored the complexities, strengths, and challenges of including clinicians and PLWHIV and HAND as peer researchers in a study of the lived experience of HIV-related neurocognitive changes.

\section{HAND: NEUROCOGNITIVE DISORDERS IN HIV}

HAND is a neurocognitive disorder linked to neuropathological changes associated with HIV (Heaton et al., 2010; Lindl, Marks, Kolson, \& Jordan-Sciutto, 2010). HAND may be classified as either Asymptomatic Neurocognitive Impairment (ANI; subtle changes in memory, attention, and/or thinking detectable using neuropsychological tests), Mild Neurocognitive Disorder (MND; mild deficits in two or more cognitive domains that are noticeable for the person experiencing them and can cause difficulty carrying out daily tasks), or HIV-Associated Dementia (HAD; severe impairment in two or more domains of cognition that causes marked difficulties in everyday functioning) (Antinori et al., 2007). Since the advent of antiretroviral therapy, the prevalence of HAD has declined, but ANI and MND continue to affect PLWHIV (Heaton et al., 2010). HIV-related cognitive changes primarily affect attention, information processing speed, memory, learning efficiency, and psychomotor skills (Akolo et al., 2014; Davis et al., 1992; Schouten, Cinque, Gisslen, Reiss, \& Portegies, 2011). Subjective reports of difficulty maintaining attention, forgetfulness, slowed cognition, and word-finding difficulties are common (Hinkin et al., 1996; Maj et al., 1994). Despite rigorous clinical studies characterizing HAND, few reports document how people living with HAND experience it in their daily lives (Gallagher et al., 2013; Hopcroft et al., 2013).

\section{HEADS UP! A CBPR APPROACH TO THE LIVED EXPERIENCE OF HAND}

Reports on the practice of involving peer researchers with cognitive issues into participatory research are few (Abma, 2018). Studies with persons living with one or more health conditions as members of their research team tend to report increased cultural and physical proximity to the health topic under investigation (Logie, James, Tharao, \& Loutfy, 2012; Muhammad et al., 2015; True, Alexander, \& Fisher, 2017), an enhanced "voice" of the patient, and greater reciprocity between roles (e.g., expert and learner) and positions in the field (e.g., insider and outsider) (Di Lorito et al., 2017). With the goal of increasing 
meaningful involvement of PLWHIV in research, PLWHIV and HIV care providers were included as study co-investigators and co-learners (Closson et al., 2016; Guta, Flicker, \& Roche, 2013; McClelland \& De Pauw, 2010) with the goal to (1) build the capacity of PLWHIV to participate in a more equitable and egalitarian way with other members of the research team, (2) build our learning on how to work with patients with complex needs, (3) facilitate participant recruitment and data collection, (4) increase the validity and reliability of our data analysis results, and (5) enhance our knowledge transfer and exchange (KTE) approaches.

CBPR, while common in HIV research (Brizay et al., 2014; Rhodes, Malow, \& Jolly, 2010), has not been used in the investigation of HAND. Furthermore, few studies of neurocognitive impairment have employed CBPR (Gallagher et al., 2013; Hopcroft et al., 2013). Our CBPR approach and outcomes can therefore help inform the process of engaging peer researchers who may be concerned about and/or experiencing cognitive impairment.

In this paper, we contribute to the literature health research involving patients by examining the strengths and challenges in the HEADS UP! Study. Specifically, we attend to the instances in which learning played a key role in resolving tensions created by the very research process. We employ a sociological lens that includes our understanding of ourselves as agents of (unintended?) change in a research setting with competing professional and lay expectations and assumptions.

\section{Objectives}

The objectives of this paper are to describe 1) how we used a CBPR approach to explore the lived experience of HAND (Methods and Activities), 2) the strengths and challenges of our CBPR approach, particularly what can be learned about the involvement of persons affected by neurocognitive issues, and 3) recommendations for involving clinicians and patients in future CBPR research in this and other areas (Results). The HEADS UP! Study results related to the experience of living with HAND are available elsewhere (Terpstra, et al., 2018).

\section{Study Methods and Activities}

We used a cross-sectional, qualitative study design to explore how PLWHIV subjectively experience, manage, and discuss HAND with others. We conducted the study at two community-based sites (Dr. Peter Centre in Vancouver, British Columbia and Casey House in Toronto, Ontario) and one hospital neuropsychology outpatient clinic (St Michael's Hospital, Toronto). We incorporated Peer Research Associate (PRA) and clinician involvement at each site.

This study was approved by the University of Toronto and St. Michael's Hospital Research Ethics Boards and the joint University of British Columbia and Providence Health Care Research Ethics Board. 
We established a community partnership and research team using existing networks. To facilitate participant, PRA, and clinician involvement, we contacted previous collaborators who were researchers and administrators at two of the community recruitment sites to be part of the research team. They then invited clinicians at these sites to be part of the research team, including a nurse and a social worker (one at each community recruitment site) who became study co-investigators. They were actively involved in every phase of the study, including participant recruitment, team meetings, data analysis and interpretation, and knowledge transfer and exchange activities.

The research team met in-person for two meetings, one held in each city where participants were recruited. The researchers also met regularly via teleconference throughout the study. In-person meetings were held to conduct group data analysis, implement KTE activities and discuss educational resource development, and manuscript preparation. (See results in Terpstra et al., 2018).

\section{Recruiting Peer Research Associates (PRAs)}

Recruitment was conducted in two of the sites dedicated exclusively to the comprehensive care of PLHIV. In Toronto, five PRAs (2 female and 3 male) were recruited from Casey House and one female PRA Engagement Coordinator, a long-time community activist in Toronto who was completing a college practicum with the NPI. The Engagement Coordinator also helped keep track of the PRAs as their participation was uneven over time. In Vancouver, at the Dr. Peter Centre, a systematic PRA selection process already existed and includes peer mentorship and support. Three PRAs (one female and two male) were hired and remained engaged until the end of the study. One of them is a co-author in this manuscript. Having neurocognitive difficulties was not a criterion to identify and recruit PRAs at either site. However, in most cases, and for different reasons, the PRAs disclosed their own struggles with memory and attention. All PRAs in this study received the same rate of compensation (thoroughly discussed by Collins et al., 2017). Of the eight PRAs who joined the study team, five (62\%) remained involved through to study completion.

Training the team. The study coordinator and the PRAs completed formal training in preparation for the study, and the entire research team engaged in ongoing learning throughout. From the start, the research team operated on the notion that all members of a team, to one degree or another had something to learn as well as offering areas of expertise. For example, all participants needed to learn about the research methods used by this study, the "technical experts" (i.e., the academic researchers) needed to brush up on published evidence and increase their awareness of the lived experience of PLWHIV and HAND while clinicians and PRAs needed to learn more about HAND and KTE. Specifically, the study coordinator was trained in qualitative research methods based on foundational readings of qualitative interviewing techniques and data analysis (Jorgensen, 1989; Rubin \& Rubin, 2011) and supervised qualitative interviews as well as data analysis in collaboration with the principal investigator and other research team members. The research coordinator was also trained and supervised in providing neuropsychological assessments and clinical interviews; this person subsequently applied to and was accepted 
into a graduate clinical psychology program and is a full member of the follow-up HEADS UP! 2 study funded at the time of writing this manuscript.

To train our PRAs, we combined the NPI's expertise in adult and distance education with subject matter proficiency in the team and the lived experience of one HIV positive community member acting as Peer Engagement Coordinator. PRAs were trained by the NPI, and study coordinator using a blended (in-person and online) learning approach (Linder, 2017). Training took approximately twenty hours over several sessions in person in Toronto and online in Vancouver. The curriculum included learning about research ethics, HAND, CBPR and the PRA roles, qualitative research methods (with a focus on coding qualitative data), and self-care. We used existing electronic learning modules available at www.uwow.ca, the training arm of the REACH collaborative research network directed by co-author Sean Rourke. PRAs were asked to complete online eModules which were discussed as a group later. Portions of the four sessions were conducted via video web conference to allow all PRAs in both cities to virtually meet and collaborate.

Recruiting study participants using HAND screening procedures. We recruited adult study participants from two community organizations, as well as one neuropsychology clinic at a large urban hospital. Clinicians who recruited participants were informed by a clinical neuropsychologist regarding signs of HAND to help them identify potential participants. The consent process required careful consideration given that invited participants were expected to be experiencing cognitive difficulties. The consent process took place in two phases, beginning with initial contact and explanation of the study by clinicians or research personnel who had pre-existing relationships with the potential participants. Clinicians went over the consent form at this initial point of contact, not to have it signed, but to make sure the consent and study process were thoroughly understood by the participants. The consent form was revisited during the initial visit with the study coordinator. Only after carefully reviewing the consent form with participants, those who decided to participate in the study were then scheduled for a neuropsychological assessment with the study coordinator. We reminded participants of their right to stop consent and withdraw without any repercussions to the services they receive at the three sites.

Eight potential participants were recruited from the hospital-based neuropsychology clinic and had already received a HAND diagnosis before being contacted about the study. Potential participants from the two other recruitment sites had not been screened for HAND and were therefore invited to participate in the neuropsychological assessment and clinical interview. The assessment was conducted by the study coordinator and lasted approximately 3.5 hours.

Nineteen of the 20 potential participants agreed to the neuropsychological assessment. After the assessment, individuals were scheduled for a feedback session with one of the two clinical neuropsychologists on the research team who summarized the results of the testing session and informed them of whether they met criteria for HAND as per current gold standard criteria (Antinori et al., 2007). The feedback session focused on participants' areas of strengths and weaknesses and suggested ways to help manage their cognitive 
difficulties. (For more details regarding the neuropsychological assessment, including the tests administered, and the feedback session, see Terpstra et al. (2018)).

Constructing and conducting qualitative interviews. Twenty-five participants were interviewed, using questions co-constructed by the principal investigator, the study coordinator and patients at one of the two community agencies and subsequently reviewed by the research team. The interview questions explored: (1) the subjective experience of cognitive impairment, (2) strategies and coping tactics used to manage cognitive impairment, and (3) the role of HAND in interactions with friends, family and healthcare providers. Sample questions included, "Are the challenges you face with memory, attention, and thinking always there?", "How do difficulties with memory influence your selfconfidence?", and "In what ways do challenges with memory, attention, or thinking affect your social life, such as the places you go or the people you spend time with?" We also administered a brief demographic questionnaire to collect information on participants' age, sex, education, recent employment history, medical history, social supports, and alcohol and drug use.

Interviews were conducted in a private room at one of the three recruitment sites by the research coordinator or principal investigator and lasted 1-2 hours. Participants were invited to skip questions, take breaks, or stop the interview at any time. They received $\$ 50$ CAD for taking part in the interview. In this approach to CBPR, peer researchers did not conduct the qualitative interviews because they were recruited from the same sites as the participants and were therefore likely to have pre-existing relationships that could violate participants' privacy and confidentiality.

Conducting participatory analysis. We adapted Flicker and Nixon's DEPICT (Dynamic reading, Engaged codebook development, Participatory coding, Inclusive reviewing and summarizing of categories, Collaborative analyzing, and Translating) model for our analysis (Flicker \& Nixon, 2015). We conducted the analysis primarily during the two inperson team meetings. We modified DEPICT as follows: First, the interviews were audio recorded and transcribed verbatim by a transcriptionist (under a confidentiality agreement). Second, the first 10 transcripts of interviews conducted in Toronto were read by at least one academic/clinician and one peer researcher and discussed at the first inperson research team meeting in Vancouver. Participant transcripts were assigned across regions and sites to prevent the unintentional identification of participants recruited from a site at which team members worked or accessed services. Third, the readers attached descriptive codes of analysis to segments of the texts. Using an iterative process, the descriptive codes were grouped into broad topic-oriented categories and the text segments belonging to the same category were compared to ensure consistency. Field notes were coded similarly. Fourth, at the second in-person meeting in Vancouver, the team members engaged in grounded theorizing (Glaser \& Strauss, 2009), which involved building consensus regarding appropriate categories to cluster closely related codes (Bryant \& Kathy, 2007; Denzin, 2006; Lewis-Beck, Bryman, \& Liao, 2003). In addition to previous qualitative research literature, our analysis and interpretations of the qualitative data were informed by clinical and lived experiences at each recruitment site. 


\section{Results: CBPR Approach involving Peers}

Although the strengths we identified carried some intrinsic challenges, we attempt to set them apart from challenges in the subsections below.

\section{STRENGTHS OF OUR CBPR APPROACH}

Insider's knowledge guided us. There is a long standing conversation about the pros and cons of peers as insiders-outsiders to a particular milieu (Burns, Fenwick, Schmied, \& Sheehan, 2012; True et al., 2017) when engaged in research and we brought this conversation to bear on our team interactions prominently featuring PRAs. Epistemologically, it has been theorized that an enquiry from the outside "is more akin to a logical positivist approach - seeking one absolute truth - whereas and enquiry from the inside more commonly echoes an interpretative approach acknowledging multiple realities" (Ritchie, Zwi, Blignault, Bunde-Birouste, \& Silove, 2009, p. 106-107). The NPI is a person living with HIV (Ibáñez-Carrasco) who has worked closely with clinicians and researchers at two of the three clinical sites, underwent screening and assessment for HAND, and received a diagnosis of mild and manageable cognitive problems somewhat typical to his age, length of time since diagnosis, and schooling/skills level. Having a person living with HIV as NPI helped ensure that the priorities of people living with HIV were constantly made visible in our research.

Those who learn together... There were two in-person team meetings that provided time for clinicians, PRAs, and researchers to become acquainted with each other and our collective approach to CBPR, and to learn from each other's unique perspectives and experiences. Our learning was reciprocal, collaborative, and continual, consistent with the model of learning alongside PRAs identified in HIV research in Canada (Caine et al., 2016; Iserbyt, 2012). PRAs added aspects of lived experience, provided real-time descriptions of HAND, and ensured thorough and thoughtful consideration of participants' perspectives even when they may not have been convenient for the researchers, clinicians, administrators, organizations, and/or institutions (e.g., that clinical environments may be slow in implementing organizational changes that are important to those accessing services and/or may have different goals/values).

Learning together also encompassed aspects of the "implicit curriculum," that is to say, learning about the "politics" of doing research in a clinical setting such as the processes involved in developing, implementing, and updating clinical research protocols in addition to relevant clinical research policies and guidelines may appear from the outside to make research and clinical uptake seem cumbersome and inefficient. For example, PRAs in our study learned the importance of doing research on HAND while at the same time they "taught" us that they experience the institutional polices discussed by health providers as limiting or even controlling. We all came to an understanding that clinicians and patients interact in institutional contexts where they have varying degrees of control and conversations between these groups are restricted. In this respect, patients and providers, when given the opportunities, are "peers" within institutional walls who have something to "teach" to each other. 
We integrated disciplinary and interpretive frames. Team members come with differing, and sometimes competing, theoretical and professional perspectives for examining how individuals and groups organize, perceive, and communicate about their reality (Charmaz, 2006); competing perspectives that at times transformed into productive tensions. For example, one of the academic researchers on the team previously formulated an episodic disability framework that we used to inform the interview schedule and data analysis (O'Brien, Bayoumi, Strike, Young, \& Davis, 2008). Based on this framework we identified a theme representing the fluctuating longitudinal course of HAND including the severity, duration, and constellations of cognitive symptoms. In another instance, community organization members of our team guided the team's attention to the comorbidities and other factors affecting the cognitive functioning of PLWHIV in addition to HAND, such as fatigue or the use of medical marihuana and relaxed our somewhat rigid initial exclusion criteria for this study to identify participants with "mostly" neurocognitive complaints.

Clinicians who worked day-to-day with people living with HAND helped us pay attention to the strategies that clinicians and people living with HAND use to manage cognitive impairment and its effects on wellbeing and health behaviours, like depressive symptoms and medical appointment attendance, respectively. Hospital-based clinicians who engaged with patients intermittently (i.e., for neuropsychological assessments and feedback sessions) were primarily interested in participants' reports regarding the assessment and diagnosis of HAND, and participants' reflections and retention regarding feedback they received about their neuropsychological functioning. Integrating these varying perspectives afforded us a more comprehensive account of participants' experiences with healthcare services aimed at addressing neurocognitive issues, such as valuable feedback regarding their ongoing support from community organizations, and how psychoeducational feedback from a neuropsychologist was received, retained, and later remembered (as discussed during the qualitative interviews).

During our qualitative data analysis sessions, PRAs highlighted descriptions of participants' and healthcare providers' limited knowledge of HAND, participants' pervasive worries about dementia, and being the recipients for even higher degrees of stigma regarding cognitive impairment. Some PRAs began to self-identify as having HAND and others vociferously rejected the label. Consequently, our data analysis was informed by diverse levels of incorporation of this "new" identity, a process that for some PLWHIV takes more time than for others (Baumgartner \& David, 2009). Even, if we theoretically knew this, we had to re-learn this lesson. Such conflicting vantage points of acceptance of the HAND label echoed the viewpoints described by study participants in the qualitative interviews, who through our study had begun to grapple with this new and potentially life-altering information. Our interview questions and responses were heavily influenced by these categories of experience, which made PRAs' diverse experiences and perspective concerning neurocognitive functioning strengths of our study.

In sum, in organic and deliberate ways, we were able to engage all research members in "frame bridging," or the "linkage of two or more ideologically congruent but structurally unconnected frames regarding a particular issue or problem" (Benford \& Snow, 2000; Snow, Rochford Jr, Worden, \& Benford, 1986, p. 467). 
We encouraged peer researchers to be KTE agents. We implemented KTE throughout the study. These activities contained the five elements (problem, context, knowledge, activities, and use) proposed by Ward, Smith, House, and Hamer (2012). In an in-person session, brainstormed on sticky notes of tips derived from participant interviews, the principal investigator, research coordinator, and PRAs drafted two brochures with "tips" for (1) health care providers and (2) patients regarding signs of HAND and how to start conversations about cognitive difficulties. Members of the team also wrote one specialized magazine article (Rourke, Terpstra, Reinhard, \& Ibáñez-Carrasco, 2015), produced one educational video about HAND (OHTN, 2015), three academic posters presented at conferences, and a video recorded public panel discussion on the role of healthcare providers in HAND-related service provision (OHTN, 2016). Using a CBPR approach to the development of KTE materials and events meant all members had greater influence than usual over the representation of the study findings. PRAs participated in videos and community events to share their experiences and knowledge related to HAND that may provide a relatable source of information for other community members as opposed to researchers without lived experience of HIV and/or HAND. In addition, PRAs' participation in the drafting and editing of KTE materials ensured that the information was conveyed in a manner deemed relevant, accessible, and interesting by people with lived experience.

\section{Challenges of our CBPR Approach}

Understanding tensions as productive learning. Recruitment, screening and assessment as well as going over consent forms and questions constitute learning processes for participants that we tend to overlook; participants get "struck" by new knowledge (Corlett, 2013). In a roundabout way, we were teaching participants living with HIV about an emerging neurocognitive concern. As highlighted later in this section, what they learn might feel validating or undermining.

During the team meetings we faced tensions between clinicians and PRAs who accessed services in one of the two community sites. We characterized this dynamic as a "productive tension," a concept consistent with those described in other patient-oriented research studies (Staley \& Barron, 2019; Witteman et al., 2018). As the in-person sessions gradually became more intimate, PRAs started to share more candid views about the experience with HIV, HAND, and the clinical services they had received at the sites. Sharing personal experiences was welcomed and allowed the team to have more insight into the lived experience of persons with HIV and possibly HAND; however, it also generated a degree of tension, a form of interpersonal and intra-organizational politics that required consideration and management. To help ensure that PRAs and clinicians felt comfortable in the research team meetings, the research coordinator consulted each PRA and clinician individually to establish how to constructively implement and maintain appropriate boundaries. These concerns and conversations demonstrate that working with peer researchers and health care providers from the same setting, while complex, can be effectively managed. It was important to acknowledge and validate each party's ideas and feedback and yet maintain focus on the objectives of our study.

Another tension emerged when a participant was diagnosed with severe neurocognitive impairment which placed the participant at risk of losing their driving license-surely an unexpected outcome. It was not our intention to bring such impact to the life of a 
participant; however, the clinicians had an ethical and legal duty to protect the public. The denouement of this situation was a verbal compromise between the patient and the clinicians and the person accepted the recommendation to cease driving. Overall, this event emphasized the careful ethical consideration necessary when a research study involves diagnosis of cognitive impairment.

The core research team (2 researchers, the NPI and the Coordinator) managed these and other tensions by acknowledging them and having private side-conversations as possible while keeping as neutral as possible. This is often perceived as what it is colloquially termed "being political" and responds to "the necessity of sustaining a Janus-faced relation with principal players" towards a productive aim (Pope, Cubellis, \& Hopper, 2016, p. 506). However, we recognize that we should have anticipated tensions and prepared for them better as underscored by a number of researchers who have looked into this matter (Boote, Baird, \& Beecroft, 2010; Duffett, 2017; Patient Engagement in Health Research: A How-to Guide for Researchers, 2018; Witteman et al., 2018).

Facing recruitment, attrition, and engagement. Living with HIV often includes experiencing several co-morbidities and being negatively impacted by various social determinants of health such as poverty, insecure housing and homelessness, and inadequate nutrition (Aidala et al., 2016; Ford et al., 2015; Worthington, Myers, O'Brien, Nixon, \& Cockerill, 2005). Furthermore, people living with HIV often face structural and systems barriers to social inclusion, including stigma and discrimination based on sexual orientation, racialized identity, or substance use. The complex realities of individuals living with HIV precluded us from recruiting HIV positive individuals with HAND and no other comorbidities - a change in our initial recruitment strategy which ultimately improved the generalizability of our findings.

PRAs were people with ties to the recruitment sites (Casey House and Dr. Peter Centre) who were aware that individuals invited to participate in the qualitative interview met criteria for HAND. Thus, if a participant told a PRA they participated in the qualitative interview (although they were interviewed by the study coordinator and NPI), the PRA could assume that the participant had met criteria for HAND. It was critical to ensure PRAs were unaware of who was participating in the study, including notifying participants of ways participation in the study could result in PRAs and others learning about their health status. However, we understood that a modicum of unintended disclosure or even gossip to could have happened among patients regardless of our best efforts and plenty of recommendations and guidelines in the field. However, gossip in healthcare facilities is being reconceptualised as "organizational communication and knowledge," (Waddington, 2016) that expresses "a range of emotions including care and concern about others, anger, annoyance and anxiety"(Waddington \& Fletcher, 2005). We learned to live with this underlying tension. This makes a research process in small health care facilities very complex and it behooves us to remaining ethical and constantly finding fresh ways of maintaining confidentiality (Ummel \& Achille, 2016).

Challenges engaging PRAs throughout this study (some of which have been flagged in literature in this field; Kilpatrick, McCartan, McAlister, \& McKeown, 2007) contributed to 
the attrition of two of eight PRAs, including lost contact (some peers live in ever-changing circumstances), deciding not to continue, and health challenges. Five PRAs remained involved until the final stages of the study, including manuscript preparation. Thus, even with the support of an HIV positive, HAND-affected Peer Engagement Coordinator to coordinate and follow-up with PRAs and providing remuneration, one female and one male PRA did not participate through to the final stages of the study.

\section{RECOMMENDATIONS}

To our knowledge, this is the first report of a CBPR approach to qualitative research in HAND that includes peer researchers living with neurocognitive challenges. In this section, we provide recommendations for conducting CBPR studies that include peer researchers living with cognitive impairment. These are predominantly derived from our experiences with managing team dynamics and engaging PRAs.

To manage sensitive boundaries. A specific recommendation resulting from our study experience concerns the recruitment of PRAs. If a PRA has been hired and more PRAs need to be recruited from that site, it can be beneficial to ask that individual to use targeted recruitment to hire one or more PRAs that already have secured a level of rapport and trust with the first PRA brought on board. This may reduce challenges to rapport and confidentiality between peer researchers previously identified in the literature (Bean \& Silva, 2010; Devotta et al., 2016) and prevent attrition, although ensuring equitable access to participation in the research enterprise for those not already connected to other peer researchers should also be considered. Additional PRA recruitment by one or more existing PRAs can help ensure a certain level of comfort between PRAs and facilitate candour and collaboration during meetings with all PRAs. Encouraging PRAs to take on this responsibility can also help distribute power (Israel, Schulz, Parker, \& Becker, 1998).

When recruiting participants and PRAs from multiple sites, conforming to each site's existing policies and procedures (policies and procedures they consider to be "tried and true") can improve efficiency and prevent tensions that may arise with uniform recruitment strategies across sites. Procedures may differ substantially from one site to the next, and by respecting the policies and procedures of the organization, there can be a greater sense of respect and trust and more familiarity for those recruiting PRAs and PRAs themselves.

PRA attrition and sporadic participation should be expected (Travers et al., 2008), potentially owing to poor health episodes (e.g., inpatient hospital stays) or a lack of trust and/or meaningful engagement. Varied opportunities that are convenient and permit intermittent involvement may help circumvent these problems. In a study that includes team members with previous clinician-client relationships, facilitating setting and monitoring boundaries between clinicians and PRAs is recommended. Including PRAs in the data analysis process, both the group discussions during in-person meetings and asking PRAs to code interviews, demonstrates that everyone provides important and valued contributions to the work. Critically, each part of the research process that included co- 
investigators also included PRAs who contributed to the research project the same as all other team members.

To sustain peer engagement. The following are four recommendations, two by the PRAs, and two by other team members:

1) PRAs recommended that future studies should invite PRAs to participate in a neuropsychological assessment prior to involvement in the study to better understand participants' experiences and, if desired, receive feedback about their cognitive functioning. The latter was particularly important to PRAs, who reported thinking to themselves, "this is me" when reading about participants' cognitive challenges. Thus, we recommend PRAs be invited to participate in the study as would a study participant, albeit with complete confidentiality, privacy, and free and informed consent. This may give PRAs even better insight into the perspectives of the participants aside from that afforded by their day-to-day lived experience (Horowitz, Robinson, \& Seifer, 2009).

2) PRAs in this study recommended additional one-on-one support for PRAs to discuss self-care and de-brief following training and full team meetings with a review of the topics, discussions, and conclusions of each. Notably, the optimal level and style of support for each PRA will differ depending on previous PRA experience (Greene, 2013). Providing a range of supports for PRAs to access and remaining sensitive and flexible to their needs, such as one-on-one debriefings after group meetings, modified training delivery methods, and frequent elicitation of feedback, are therefore recommended.

3) PRAs may find participants' descriptions of cognitive difficulties overwhelming, particularly if those descriptions resemble their own experiences. In our study, some of the peer researchers had been diagnosed with HAND, and others had not previously been assessed. Conversations with each group of PRAs about analyzing data on a subject that personally relates to them, and the emotional and social impact the process may have, is recommended in preparation for qualitative analysis of interviews to provide a safe space to reflect on and process their experience. These conversations may include, for example, tailored self-care procedures that support them as they anticipate, discuss, and remember the interviews they analyzed, particularly through open discussion and validation of their experiences.

4) Special attention should be paid to the unnecessary use of scientific jargon (Boote et al., 2010). All team members should use accessible language that promotes full and unrestricted involvement throughout the study. In the training, ample space should be allocated for PRAs to understand necessary jargon such as HAND and what it means to them. This also addresses "knowledge dominance" in HIV research which prevents peer researchers from participating fully in the research process. For example, fear of appearing ignorant or falling behind in the conversations and subsequently disengaging (Wallerstein \& Duran, 2010). Clarifying and exploring research terms and concepts can help PRAs develop expertise and prevent drop-out (Ahmed \& Palermo, 2010). 
To include peers in analytical and KTE stages of the research study. Although uncommon in CBPR (Flicker \& Nixon, 2014, 2004), giving PRAs the opportunity to analyze data and help prepare manuscripts can promote engagement throughout the course of the study by making the work more meaningful for them (Ahmed \& Palermo, 2010). It reinforces the principle that all team members provide valuable expertise and insights no matter the stage of the study (Israel et al., 1998; Wallerstein \& Duran, 2010). Furthermore, the expertise that PRAs bring, specifically lived experience of certain aspects of the phenomenon being studied, helps to ground the data in the perspectives of individuals with similar experience (Minkler, 2005) and in doing so can reduce bias in the interpretation of the data improve the study's external validity (Ahmed \& Palermo, 2010; Horowitz et al., 2009; Israel et al., 1998). One caveat is to (over?) prepare at the start of the study for contingencies. We missed a few steps such as including our PRAs in the screening and assessment of HAND. Another caveat is that team will often encounter structural barriers to including PRAs and participants more fully this. For example, KTE often happens, or continues, well beyond the funding timeline of the grant. It is difficult to compensate peers for such activities. We need funding agencies to provide additional funds, opportunities and considerations for supporting these activities.

\section{ConCLUSIONS}

A CBPR approach was integral to our study exploring the experiences of living with HAND. Strengths of our CBPR approach included an interdisciplinary team encompassing diverse areas of research and clinical expertise and the involvement of PRAs in data analysis and interpretation-strengths that represent practical operationalization of a globally subscribed principle of meaningful inclusion of people living with HIV in activities such as research that directly impact them (The GIPA principle - reference). However, the teaching and learning necessary were not always equitable and well-distributed, a fact we will face better in the funded follow-up larger study. Challenges included navigating the collaboration of clinicians and PRAs with previous clinician-patient relationships, problemsolving in response to participant recruitment dilemmas, keeping PRAs engaged throughout the course of the study, and the additional time, effort, and resources required for a reciprocal learning approach. The outcomes of our study suggest that engaging an interdisciplinary team across multiple sites with PRAs is a valuable method for comprehensively exploring the lived experience of a complex chronic condition such as HAND. This CBPR approach may be useful to people living with chronic illness, community organizations, researchers, and clinicians when engaging in participatory research that leads to action.

\section{ACKNOWLEDGEMENTS}

This work was supported by a Canadian Institutes of Health Research (CIHR) Catalyst Grant and REACH 2.0. KOB is supported by a New Investigator Award from CIHR. In memory of Victor T., a PRA on the project. 


\section{REFERENCES}

Abma, T. A. (2018). Dialogue and deliberation: New approaches to including patients in setting health and healthcare research agendas. Action Research, 1476750318757850 .

Ahmed, S. M., \& Palermo, A.-G. S. (2010). Community engagement in research: Frameworks for education and peer review. American Journal of Public Health, 100(8), 13801387.

Aidala, A. A., Wilson, M. G., Shubert, V., Gogolishvili, D., Globerman, J., Rueda, S., . . Rourke, S. B. (2016). Housing status, medical care, and health outcomes among people living with HIV/AIDS: A systematic review. American Journal of Public Health, 106(1), e1e23.

Akolo, C., Royal III, W., Cherner, M., Okwuasaba, K., Eyzaguirre, L., Adebiyi, R., . . Blattner, W. A. (2014). Neurocognitive impairment associated with predominantly early stage HIV infection in Abuja, Nigeria. Journal of Neurovirology, 20(4), 380-387.

Antinori, A., Arendt, G., Becker, J., Brew, B., Byrd, D., Cherner, M., . . Goodkin, K. (2007). Updated research nosology for HIV-associated neurocognitive disorders. Neurology, 69(18), 1789-1799.

Baumgartner, L., \& David, K. (2009). Accepting being poz: The incorporation of the HIV identity into the self. Qualitative Health Research, 19(12), 1730-1743. doi:10.1177/1049732309352907

Bean, S., \& Silva, D. S. (2010). Betwixt \& between: Peer recruiter proximity in communitybased research. The American Journal of Bioethics, 10(3), 18-19.

Benford, R. D., \& Snow, D. A. (2000). Framing processes and social movements: An overview and assessment. Annual Review of Sociology, 26(1), 611-639.

Boote, J., Baird, W., \& Beecroft, C. (2010). Public involvement at the design stage of primary health research: A narrative review of case examples. Health Policy, 95, 10-23.

Brizay, U., Golob, L., Globerman, J., Gogolishvili, D., Bird, M., Rios-Ellis, B., . . Heidari, S. (2014). Community-academic partnerships in HIV-related research: A systematic literature review of theory and practice. Journal of the International AIDS Society, 18(1), 19354-19354.

Bryant, A., \& Kathy, C. (2007). The SAGE Handbook of Grounded Theory. London: SAGE.

Burns, E., Fenwick, J., Schmied, V., \& Sheehan, A. (2012). Reflexivity in midwifery research: The insider/outsider debate. Midwifery, 28(1), 52-60. 
Caine, V., Mill, J., O'brien, K., Solomon, P., Worthington, C., Dykeman, M., . . Arneson, C. (2016). Implementation process of a Canadian community-based nurse mentorship intervention in HIV care. Journal of the Association of Nurses in AIDS Care, 27(3), 274-284.

Charmaz, K. (2006). Constructing grounded theory: A practical guide through qualitative analysis. Thousand Oaks, California: SAGE.

Closson, K., McNeil, R., McDougall, P., Fernando, S., Collins, A., Turje, R. B., . . Parashar, S. (2016). Meaningful engagement of people living with HIV who use drugs: Methodology for the design of a Peer Research Associate (PRA) hiring model. Harm Reduction Journal, 13(1), 26.

Collins, A. B., Strike, C., Guta, A., Turje, R. B., McDougall, P., Parashar, S., \& McNeil, R. (2017). "We're giving you something so we get something in return": Perspectives on research participation and compensation among people living with HIV who use drugs. International Journal of Drug Policy, 39, 92-98.

Corlett, S. (2013). Participant learning in and through research as reflexive dialogue: Being 'struck' and the effects of recall. Management Learning, 44(5), 453-469. doi:10.1177/1350507612453429

Cummins, D., Waters, D., Aggar, C., Crawford, D., Fethney, J., \& O’Connor, C. (2018). Voices from Australia-concerns about HIV associated neurocognitive disorder. AIDS Care, 1-9. doi:10.1080/09540121.2018.1426826

Davis, L., Hjelle, B., Miller, V., Palmer, D., Llewellyn, A., Merlin, T., ... Wiley, C. (1992). Early viral brain invasion in iatrogenic human immunodeficiency virus infection. Neurology, 42(9), 1736-1736.

Denzin, N. (2006). Sociological methods: A sourcebook (5th ed.). New Brunswick, N.J: Aldine Transaction.

Devotta, K., Woodhall-Melnik, J., Pedersen, C., Wendaferew, A., Dowbor, T. P., Guilcher, S. J., . . . Matheson, F. I. (2016). Enriching qualitative research by engaging peer interviewers: A case study. Qualitative Research, 16(6), 661-680.

Di Lorito, C., Birt, L., Poland, F., Csipke, E., Gove, D., Diaz-Ponce, A., \& Orrell, M. (2017). A synthesis of the evidence on peer research with potentially vulnerable adults: How this relates to dementia. International Journal of Geriatric Psychiatry, 32(1), 58-67.

Duffett, L. (2017). Patient engagement: What partnering with patient in research is all about. Thrombosis Research, 150, 113-120. 
Eaton, A. D. M. R., Craig, S. L. P. R. L., \& Wallace, R. M. R. (2017). The intersecting cognitive and aging needs of HIV-positive older adults: Implications for social work practice. Social Work Health Care, 56(8), 733-747. doi:10.1080/00981389.2017.1339759

Flicker, S., \& Nixon, S. A. (2014). The DEPICT model for participatory qualitative health promotion research analysis piloted in Canada, Zambia and South Africa. Health Promotion International, 30(3), 616-624.

Flicker, S., \& Nixon, S. A. (2015). The DEPICT model for participatory qualitative health promotion research analysis piloted in Canada, Zambia and South Africa. Health Promotion International, 30(3), 616-624.

Ford, N., Shubber, Z., Meintjes, G., Grinsztejn, B., Eholie, S., Mills, E. J., . . Nsanzimana, S. (2015). Causes of hospital admission among people living with HIV worldwide: a systematic review and meta-analysis. The Lancet HIV, 2(10), e438-e444.

Gallagher, S., Biro, S., Creamer, E., Della Rossa, E., Collins, E., Rourke, S., \& Nixon, S. (2013). "It's a hidden Issue": Exploring the experiences of women with HIV-associated neurocognitive challenges using a disability framework. Disability and Rehabilitation, 35(1), 36-46.

Glaser B. G., Strauss A. L. (2009). The discovery of grounded theory: Strategies for qualitative research. Chicago: Aldine Transaction.

Greene, S. (2013). Peer research assistantships and the ethics of reciprocity in communitybased research. Journal Empirical Research on Human Research Ethics, 8(2), 141152. doi:10.1525/jer.2013.8.2.141

Guta, A., Flicker, S., \& Roche, B. (2013). Governing through community allegiance: A qualitative examination of peer research in community-based participatory research. Critical Public Health, 23(4), 432-451.

Heaton, R., Clifford, D., Franklin, D., Woods, S., Ake, C., Vaida, F., . . Atkinson, J. (2010). HIVassociated neurocognitive disorders persist in the era of potent antiretroviral therapy CHARTER Study. Neurology, 75(23), 2087-2096.

Hinkin, C. H., van Gorp, W. G., Satz, P., Marcotte, T., Durvasula, R. S., Wood, S., ... Baluda, M. R. (1996). Actual versus self-reported cognitive dysfunction in HIV-1 infection: memory-metamemory dissociations. Journal of Clinical and Experimental Neuropsychology, 18(3), 431-443.

Hopcroft, L., Bester, L., Clement, D., Quigley, A., Sachdeva, M., Rourke, S. B., \& Nixon, S. A. (2013). "My body's a 50 year-old but my brain is definitely an 85 year-old": Exploring the experiences of men ageing with HIV-associated neurocognitive challenges. Journal of the International AIDS Society, 16(1), 18506. 
Horowitz, C. R., Robinson, M., \& Seifer, S. (2009). Community-based participatory research from the margin to the mainstream are researchers prepared? Circulation, 119(19), 2633-2642.

Iserbyt, P. (2012). Reciprocal Learning. In N. M. Seel (Ed.), Encyclopedia of the Sciences of Learning (pp. 2785-2786). Boston, MA: Springer US.

Israel, B. A., Schulz, A. J., Parker, E. A., \& Becker, A. B. (1998). Review of community-based research: Assessing partnership approaches to improve public health. Annual Review of Public Health, 19(1), 173-202.

Jorgensen, D. L. (1989). Participant observation: A methodology for human studies. Applied social research methods series, Vol. 15. Thousand Oaks, CA: SAGE. http://dx.doi.org/10.4135/9781412985376

Kilpatrick, R., McCartan, C., McAlister, S., \& McKeown, P. (2007). 'If I am brutally honest, research has never appealed to me...' The problems and successes of a peer research project. Educational Action Research, 15(3), 351-369.

Lewis-Beck, M. S., Bryman, A., \& Futing Liao, T. (2004). The SAGE encyclopedia of social science research methods. Thousand Oaks, CA: SAGE. doi: 10.4135/9781412950589

Linder, K. E. (2017). Fundamentals of Hybrid teaching and learning. New Directions for Teaching and Learning, 2017(149), 11-18.

Lindl, K. A., Marks, D. R., Kolson, D. L., \& Jordan-Sciutto, K. L. (2010). HIV-Associated Neurocognitive Disorder: Pathogenesis and therapeutic opportunities. Journal of Neuroimmune Pharmacology, 5(3), 294-309. doi:10.1007/s11481-010-9205-z

Logie, C., James, L., Tharao, W., \& Loutfy, M. R. (2012). Opportunities, ethical challenges, and lessons learned from working with peer research assistants in a multi-method HIV community-based research study in Ontario, Canada. Journal of Empirical Research on Human Research Ethics, 7(4), 10-19. doi:10.1525/jer.2012.7.4.10

Maj, M., Satz, P., Janssen, R., Zaudig, M., Starace, F., D'Elia, L., . . Ndetei, D. (1994). WHO Neuropsychiatric AIDS Study, cross-sectional phase II: Neuropsychological and neurological findings. Archives of General Psychiatry, 51(1), 51-61.

McClelland A., \& De Pauw L. (2010). Greater Involvement of people living with HIV (GIPA): Good Practice Guide. Amsterdam, Netherlands: International HIV/AIDS Alliance and the Global Network of People Living with HIV (GNP+).

McDermott, A., Zaporojan, L., McNamara, P., Doherty, C. P., Redmond, J., Forde, C., ... Bergin, C. (2017). The effects of a 16-week aerobic exercise programme on cognitive function in people living with HIV. AIDS Care, 29(6), 667-674. doi:10.1080/09540121.2016.1263723 
Mendes, A. (2015). Contending with the chronicity of HIV and associated comorbidities. British Journal of Community Nursing, 20(11), 568. doi:10.12968/bjcn.2015.20.11.568

Minkler, M. (2005). Community-based research partnerships: Challenges and opportunities. Journal of Urban Health, 82(2), ii3-ii12.

Muhammad, M., Wallerstein, N., Sussman, A. L., Avila, M., Belone, L., \& Duran, B. (2015). Reflections on researcher identity and power: The impact of positionality on community based participatory research (CBPR) processes and outcomes. Critical Sociology, 41(7-8), 1045-1063.

O'Brien, K. K., Bayoumi, A. M., Strike, C., Young, N. L., \& Davis, A. M. (2008). Exploring disability from the perspective of adults living with HIV/AIDS: Development of a conceptual framework. Health and Quality of Life Outcomes, 6(1), 76. doi:10.1186/1477-7525-6-76

Ontarui HIV Treatment Network (OHTN). (2015). Claudia: HEADS UP! Retrieved from https://www.youtube.com/watch?v=R0c 0penI30

Ontario HIV Treatment Netword (OHTN). (2016). Café Scientifique: All about H.A.N.D. Retrieved from https://www.youtube.com/watch?v=BFc1Ugha6aY\&feature=emupload owner

Patient Engagement in Health Research: A How-to Guide for Researchers. (2018). Retrieved from https://albertainnovates.ca/wp-content/uploads/2018/06/How-To-GuideResearcher-Version-8.0-May-2018.pdf

Pope, L. G., Cubellis, L., \& Hopper, K. (2016). Signing on for dirty work: Taking stock of a public psychiatry project from the inside. Transcultural Psychiatry, 53(4), 506-526. doi:10.1177/1363461516655947

Rhodes, S. D., Malow, R. M., \& Jolly, C. (2010). Community-based participatory research (CBPR): A new and not-so-new approach to HIV/AIDS prevention, care, and treatment. AIDS Education and Prevention, 22(3), 173.

Ritchie, J., Zwi, A. B., Blignault, I., Bunde-Birouste, A., \& Silove, D. (2009). Insider-outsider positions in health-development research: Reflections for practice. Development in Practice, 19(1), 106-112.

Rourke, S. B., Terpstra, A. R., Reinhard, R., \& Ibáñez-Carrasco, F. (Spring 2015). Mild brain injuries in HIV often go unrecognized - more needs to be done. IMS Magazine, Spring 2015, 4. 
Rubin H. J., \& Rubin, Irene S. (2003). Qualitative interviewing (2nd ed.): The art of hearing data. Thousand Oaks, CA: SAGE.

Rubin, H. J., \& Rubin, I. S. (2011). Qualitative interviewing: The art of hearing data. SAGE.

Schouten, J., Cinque, P., Gisslen, M., Reiss, P., \& Portegies, P. (2011). HIV-1 infection and cognitive impairment in the cART era: A review. Aids, 25(5), 561-575.

Snow, D. A., Rochford Jr, E. B., Worden, S. K., \& Benford, R. D. (1986). Frame alignment processes, micromobilization, and movement participation. American Sociological Review, 464-481.

Staley, K., \& Barron, D. (2019). Learning as an outcome of involvement in research: What are the implications for practice, reporting and evaluation? Research Involvement and Engagement, 5(1), 14. doi:10.1186/s40900-019-0147-1

Tedaldi, E. M., Minniti, N. L., \& Fischer, T. (2015). HIV-associated neurocognitive disorders: The relationship of HIV infection with physical and social comorbidities. Biomed Research Internation, 2015. doi:10.1155/2015/641913

Terpstra, A. R., Worthington, C., Ibáñez-Carrasco, F., O’Brien, K. K., Yamamoto, A., Chan Carusone, S., . . Rourke, S. B. (2018). "I'm just forgetting and I don't know why": Exploring how people living with HIV-associated neurocognitive disorder view, manage, and obtain support for their cognitive difficulties. Qualitative Health Research, 28(6), 859-872.

Travers, R., Wilson, M. G., Flicker, S., Guta, A., Bereket, T., McKay, C., . . Globerman, J. (2008). The greater involvement of people living with AIDS principle: Theory versus practice in Ontario's HIV/AIDS community-based research sector. AIDS Care, 20(6), 615-624.

True, G., Alexander, L. B., \& Fisher, C. B. (2017). Supporting the role of community members employed as research staff: Perspectives of community researchers working in addiction research. Social Science \& Medicine, 187, 67-75.

Ummel, D., \& Achille, M. (2016). How Not to Let Secrets Out When Conducting Qualitative Research With Dyads. Qualitative Health Research, 26(6), 807-815. doi:10.1177/1049732315627427

Waddington, K. (2016). Rethinking gossip and scandal in healthcare organizations. Journal of Health Organization and Management, 30(6), 810-817. doi:http://dx.doi.org.myaccess.library.utoronto.ca/10.1108/JHOM-03-2016-0053

Waddington, K., \& Fletcher, C. (2005). Gossip and emotion in nursing and health-care organizations. Journal of Health Organization and Management, 19(4/5), 378-394. doi:10.1108/14777260510615404 
Wallerstein, N., \& Duran, B. (2010). Community-based participatory research contributions to intervention research: The intersection of science and practice to improve health equity. American Journal of Public Health, 100(S1), S40-S46.

Ward, V., Smith, S., House, A., \& Hamer, S. (2012). Exploring knowledge exchange: A useful framework for practice and policy. Social Science \& Medicine, 74(3), 297-304. doi:10.1016/j.socscimed.2011.09.021

Witteman, H. O., Chipenda Dansokho, S., Colquhoun, H., Fagerlin, A., Giguere, A. M. C., Glouberman, S., ... Volk, R. J. (2018). Twelve lessons learned for effective research partnerships between patients, caregivers, clinicians, academic researchers, and other stakeholders. Journal of General Internal Medicine. doi:10.1007/s11606-0174269-6

Worthington, C., Myers, T., O'Brien, K., Nixon, S., \& Cockerill, R. (2005). Rehabilitation in HIV/AIDS: Development of an expanded conceptual framework. AIDS Patient Care and STDs, 19(4), 258-271.

\section{BIOGRAPHICAL NOTE:}

Dr. Francisco Ibáñez-Carrasco, Senior Research Associate, Centre for Urban Health Solutions, St Michael's Hospital.

Alex R. Terpstra, MSc, Department of Psychology, University of British Columbia.

Dr. Sean Rourke, Scientist, Li Ka Shing Knowledge Institute, St. Michael’s Hospital.

Dr. Aiko Yamamoto, Department of Psychiatry, University of British Columbia.

Dr. Soo Chan Carusone, Director of Research, Casey House, Toronto.

Rosalind Baltzer-Turje, Senior Director of Clinical Programs, Dr. Peter AIDS Foundation.

Patrick McDougall, Director of Knowledge Translation and Evaluation, Dr. Peter AIDS Foundation.

Claudia Medina, Program Manager, PASAN Toronto.

Dr. Kelly K. O’Brien, Associate Professor, Department of Physical Therapy, University of Toronto.

Dr. Catherine Worthington, Professor and Director, School of Public Health and Social Policy, University of Victoria, British Columbia. 